\title{
La formación de la cultura ambiental en el estudiante de la carrera de Derecho*
}

\author{
Alcides Antúnez Sánchez ${ }^{* *}$ \\ Elena López Espinosa****
}

Recibido: 3 de febrero de 2019 • Aprobado: 5 de marzo de 2019

\section{Resumen}

El artículo presenta una mirada a la formación jurídica ambiental del estudiante de Derecho, cuya adecuada ejecución incidirá en el conocimiento de la educación ambiental para alcanzar el desarrollo sostenible, en el que la relación hombre-naturaleza, y a fin de lograr la conservación de las condiciones ambientales que requiere para su hábitat sin perjuicio del desarrollo de las actividades económicas que lo impactan, necesita establecer los mecanismos, las medidas y los procedimientos que permitan una convivencia armónica de lo natural, de lo social y de lo económico debido a la transdisciplinariedad de la cuestión ambiental. Se propone como objetivo entonces fomentar el proceso de formación jurídica ambiental en el estudiante de la carrera de Derecho, a partir de la teoría y la praxis, que permita educar un profesional con competencias a causa de la transdisciplinariedad de la cuestión ambiental incidente

* Este artículo es producto del proyecto de investigación "La formación de la cultura ambiental en el estudiante de la carrera de Derecho", gestionado en la Universidad de Valencia, España. Citar como: Antúnez Sánchez, A. y López Espinosa, E. (2019). La formación de la cultura ambiental en el estudiante de la carrera de Derecho. Revista IUSTA, 51(2), 61-89. DOI: https://doi. org/10.15332/25005286.5033

** Magíster en Asesoría Jurídica por la Universidad de Oriente de Cuba. Profesor de la Universidad de Granma, Bayama, Cuba. Correo electrónico: aantunez@udg.co.cu

*** Licenciada en Pedagogía por la Universidad de Granma, Bayama, Cuba. Correo electrónico: elopez@udg.co.cu 
en el desarrollo sostenible. Para ello, se utilizaron los métodos de análisis síntesis, inducción-deducción, teórico-jurídico e histórico lógico.

Palabras clave: competencias, conciencia ambiental, cultura ambiental, formación TIC.

\title{
THE FORMATION OF THE ENVIRONMENTAL CULTURE IN THE LAW STUDENT
}

\begin{abstract}
The article presents a look at the environmental legal education of the law student, its proper execution will influence the knowledge of environmental education to achieve sustainable development, where man-nature and in order to achieve the preservation of the environmental conditions required for its habitat without prejudice to the development of the economic activities that affect it, needing to establish the mechanisms, measures and procedures that enable a harmonious coexistence of the natural, of the social and of the economic for its transdisciplinarity of the environmental issue. It proposes as objective to argue the need to promote in the process of environmental legal education in the law student, from the theory and practice, to accomplish a professional with competencies due to the transdisciplinarity of the environmental issue incidental in sustainable development. To do so, the analysis synthesis, deduction induction, legal-theoretical, and logical history methods are used.
\end{abstract}

Keywords: competencies, environmental awareness, environmental culture, ICT training.

\section{A formação da Cultura ambiental no estudante da GRAduAÇÃo De Direito}

\section{Resumo}

O manuscrito apresenta um olhar da formação jurídica ambiental do estudante de Direito, sua adequada execução incidirá no conhecimento da educação ambiental para atingir o desenvolvimento sustentável, onde o homem-natureza e com o objetivo de lograr a conservação das condições ambientais que requer para 
seu habitat sem prejuízo do desenvolvimento das atividades econômicas que o impactam, necessitada de estabelecer os mecanismos, medidas e procedimentos que permitam uma convivência harmônica do natural, do social e do econômico pela sua transdisciplinariedade da questão ambiental. Propõe-se como objetivo argumentar a necessidade de fomentar no processo de formação jurídica ambiental no estudante da graduação de Direito, a partir da teoria e da práxis, que permita atingir um profissional com competências pela transdisciplinariedade da questão ambiental incidente no desenvolvimento sustentável. Para isso são utilizados os métodos de analise síntese, indução dedução, teórico-jurídico e o histórico lógico.

Palavras-chave: competências, consciência ambiental, cultura ambiental, formação TIC.

\section{Introducción}

La protección del medio ambiente es una cuestión crucial para la supervivencia de la especie humana y de toda forma de vida en la Tierra. Ello es una prioridad insoslayable en la formulación de las políticas públicas y en la elaboración del ordenamiento jurídico de un país para lograr alcanzar el desarrollo sostenible en el siglo xxi. Por ello, la irresponsabilidad de atentar contra los bienes jurídicos protegidos por el Estado debe tener un límite: quien incurra en conductas antijurídicas puede ser objeto de aplicación de sanciones administrativas, civiles y penales por la comisión de delitos ambientales; al ritmo que vamos, aun cuando los derechos de la naturaleza estén en la categoría de los derechos humanos y por tanto sujetos a tutela judicial, a corto y mediano plazo será irreversible reparar el daño causado, cuyos efectos no se harán esperar, acrecentados en este siglo.

La educación ambiental es un instrumento fundamental para alcanzar los grandes objetivos necesarios para un desarrollo sustentable o sostenible, el cual propone que la población adquiera conocimientos acerca de los aspectos naturales, culturales y sociales, y que contribuyen a la solución de los problemas ambientales, a la vez que vincula al ser humano con su entorno. La educación ambiental propicia un cambio de actitudes y una participación responsable en la gestión del medio y crea actuaciones adecuadas con el entorno natural. Además, fomenta el compromiso para contribuir al cambio social, cultural y económico, a partir del desarrollo de un amplio abanico de valores, actitudes y habilidades que le permita a cada persona 
formarse criterios propios, asumir su responsabilidad general y desempeñar un papel constructivo.

Es reconocido por científicos que el problema ambiental en la actualidad es complejo, pues no se ha logrado alcanzar el equilibrio necesario entre el desarrollo económico y la explotación racional de los ecosistemas en los que se encuentran los recursos naturales, al verse este fragmentado y no de forma integrada en su solución. Se propone para ello como objetivo fomentar el proceso de formación jurídico-ambiental en el estudiante de la carrera de Derecho, a partir de la teoría y la praxis, con el que se permita alcanzar un profesional con competencias debido a la transdisciplinariedad de la cuestión ambiental incidente en el desarrollo sostenible.

\section{La formación de la cultura ambiental del estudiante de la carrera de Derecho}

El proceso de formación de la cultura ambiental del estudiante de la carrera de Derecho en Cuba es considerado como una dimensión de la formación integral de este profesional, como el proceso que parte de la consideración de que en una sociedad democrática, desde el sentido primogénito de la justicia, la equidad, la solidaridad y la tolerancia a la diversidad, que aspira a elevar su cultura ética de un nivel empírico, y de sentido común, a un nivel de reflexión argumentativa y justificativa, por lo que cobra especial relevancia en este contexto el abordaje de las causas de la crisis ambiental actual: el modo de relación establecido por la sociedad industrial globalizada con el mundo natural y las consecuencias adversas que genera el cambio climático en el siglo xxi.

Esta formación ambiental guarda relación con los documentos aprobados en la Organización de las Naciones Unidas para la Educación, la Ciencia y la Cultura (Unesco), con el Decenio de las Naciones Unidas de la Educación para el Desarrollo Sostenible (2005-2014): plan de aplicación internacional, en el que el subsistema de reconocimiento ambiental profesional condiciona la realización de la educación en valores ambientales profesionales, como el proceso práctico que materializa a partir de los subsistemas de valoración ambiental profesional y de actuación ambiental profesional las propuestas de prevención o mitigación de los problemas ambientales en la concepción "pensar globalmente y actuar localmente".

La Unesco y el Programa de las Naciones Unidas para el Medio Ambiente (PNUMA) registran en su haber la creación de la Comisión de Educación como los 
antecedentes del proceso a través del cual se fue construyendo a nivel internacional la percepción sobre la necesidad de incorporar la dimensión ambiental y su naturaleza de carácter interdisciplinario en las universidades como entes formadores (Barragán, 2016).

En el PNUMA, se estableció como el principal programa de la Organización de las Naciones Unidas (ONU) encargado de los temas ambientales, con la misión de dirigir y alentar la participación en el cuidado del medio ambiente, para inspirar, informar y dar a las naciones y a los pueblos los medios de mejorar la calidad de vida sin poner en peligro la de las futuras generaciones. De aquí la necesidad de que la cuestión ambiental sea un tema pertinente en las universidades en el proceso formativo, para lo cual reseñó que

las políticas de economía ambiental en el contexto del desarrollo sostenible y la erradicación de la pobreza deberán ser compatibles con el Derecho Internacional; respetar la soberanía nacional de cada país sobre sus recursos naturales, al tener en cuenta sus circunstancias, objetivos, responsabilidades, prioridades y margen de acción con respecto a las tres dimensiones del desarrollo sostenible. (Barragán, 2016, p. 45)

Por ello, se justiprecia que la educación jurídico-ambiental debe desarrollarse permeando la misión de la universidad, resguardando, divulgando o desarrollando la cultura jurídico-ambiental, y no quedarse el jurista solo vinculado a las leyes, según se dispone en el capítulo 36 de la Agenda 21. Ello explica, a partir de lo apreciado por los autores de este artículo, por qué se puede distinguir un gran número de corrientes en el campo de la educación ambiental, enfocada en el paradigma que asuma el concepto de sistema para comprender y proyectar de manera sostenible la relación hombre-naturaleza como reto que enfrenta la educación en lograr un enfoque inter y transdisciplinario que permita articular los saberes ambientales.

La toma de conciencia que se advierte hoy en el mundo sobre la cuestión ambiental es la respuesta a lo que el mundo se enfrenta, a causa del olvido centenario que hicimos como integrantes del todo. No se puede perder de vista el lugar que ocupamos como un componente más del medio ambiente, debido a los impactos que estamos en capacidad de efectuar en él, de ahí la necesidad de una formación de una cultura ambiental que permita dar respuestas adecuadas para su mitigación o solución desde su modo de actuación. 
Son analizados en este sentido los aportes de Leff (2001), centrados en la epistemología ambiental y la ecología política, dentro de los procesos institucionales y movimientos sociales en favor de la educación ambiental en América Latina y el Caribe, en los que se hace un estudio de la complejidad ambiental desde una perspectiva crítica y analítica, que rebasa los discursos políticos.

Celeiro (2013), quien considera que la moral y la ética no nacen con el hombre, como cristalizaciones y productos culturales no están encarnados en él, sino en el mundo que los rodea; de esta forma, solo en el proceso de asimilación él adquiere los conocimientos, las capacidades, las habilidades y que convierte en propios, y esto es posible cuando entra en relación con el mundo a través de otras personas, lo cual, según Leontiev (1966), es un proceso de educación.

Díaz (2011) considera que el Derecho como totalidad compleja, se configura a partir de ramas independientes del Derecho, que se excluyen y se complementan, se excluyen en tanto cada una de esas ramas posee un objeto específico de protección y se complementan en tanto no pueden ser advertidas sino en relación con las otras ramas y contenidos vinculantes, que pueden encontrarse incluso fuera de las disciplinas jurídicas, evidenciando el carácter totalizador del Derecho y su estructura tridimensional, como ciencia, como rama y como atributo de la personalidad. (p. 89)

Por esta razón, Pérez-Jorge, Barragán-Medero y Molina-Fernández (2017) refieren que la educación ambiental, al ser básicamente una cuestión actitudinal, se enfrenta a uno de los más difíciles problemas didácticos: ¿cómo se aprenden los valores?, ¿cómo se cambia de actitud? No se trata de adoctrinar, de imponer los valores, sino de situar al alumnado en condiciones de reflexionar y descubrir un sistema propio y adecuado.

Dentro del ordenamiento jurídico cubano, en relación con lo antes abordado, en el texto constitucional, desde el artículo 27 , se regula:

E1 Estado protege el medio ambiente y los recursos naturales del país. Reconoce su estrecha vinculación con el desarrollo económico y social sostenible para hacer más racional la vida humana y asegurar la supervivencia, el bienestar y la seguridad de las generaciones actuales y futuras. Corresponde a los órganos competentes aplicar esta política. Es deber de los ciudadanos contribuir a la protección del agua, la 
atmósfera, la conservación del suelo, la flora, la fauna y todo el rico potencial de la naturaleza.

A tales efectos, el Ministerio de Ciencia, Tecnología y Medio Ambiente (cITMA), como organismo de la Administración pública, establece las coordinaciones correspondientes con el Ministerio de Educación Superior y el Ministerio de Cultura, con los medios de difusión, otros órganos y organismos. El artículo 50 de la Ley 81/1997 dispone que

el Ministerio de Educación Superior garantizará la introducción de la dimensión ambiental, a partir de los modelos del profesional y de los planes de estudios de pre y postgrado y de extensión y actividades docentes y extradocentes, dirigidas a la formación y el perfeccionamiento de los profesionales de todas las ramas.

En el ordenamiento jurídico cubano, se aprecia cómo la Estrategia Ambiental Nacional (2016-2020) recoge los principales problemas ambientales de la nación, relacionados con "la degradación de los suelos, las afectaciones a la cobertura forestal, la pérdida de la biodiversidad biológica y de los ecosistemas, la contaminación, el uso del agua, y el cambio climático". ${ }^{1}$

Este acto administrativo toma como punto de partida los problemas ambientales presentes en las diferentes áreas en Cuba como resultado del diagnóstico ambiental, las fortalezas y las debilidades que le permiten insertarse a través de diferentes vías en la solución de los problemas ambientales, expresado en el enunciado de su misión.

Para su contextualización, la Estrategia Ambiental Nacional (2016-2020) emerge como el documento público en el que se dispone que "en la Educación Superior también se vienen haciendo esfuerzos, sobre todo en las carreras cuyos perfiles están vinculados a los recursos naturales, y en particular en algunos centros de formación de docentes y se han obtenido significativos resultados en la investigación vinculada a la solución de problemas ambientales".

Nos indica que la universidad cubana tiene como objeto de estudio incidir en los procesos de formación jurídico-ambientales del profesional, teniendo en cuenta que para que realmente se propicie el cambio de paradigma hacia el desarrollo

1 Este acto administrativo aún no ha sido publicado en la Gaceta Oficial de la República de Cuba, estando en vigencia la correspondiente al ciclo 2010-2015, elaborada por el cITMA. 
sostenible no basta con que esta retome su papel de vanguardia en la generación de pensamiento y en la formación de profesionales abocados a la solución de los problemas de la sociedad, lo que implica que se realicen transformaciones internas de índole organizacional y de diseño institucional.

En este sentido, la concepción que se presenta en este trabajo se sustenta en fundamentos epistemológicos de esencia ética, sociológica, jurídica y psicopedagógica, que resultan pertinentes y necesarios para el nuevo constructo que se argumenta en el proceso de formación del estudiante de la carrera de Derecho. Los referentes teóricos asumidos como fundamentos se detallan a continuación.

Desde lo ético, los presupuestos de la ética de orientación dialéctico-materialista concretados en los principios metodológicos de la unidad de la conciencia moral y la conducta, la correlación entre el deber ser y el ser como especificidad de la regulación moral, elementos que se significan en esta investigación. La indisoluble unidad de lo objetivo y lo subjetivo, lo material y lo ideal, aparece visiblemente en el hombre en tanto se le toma como sujeto de la actividad; o sea, en la actividad humana aparecen en unidad los componentes objetivos, materiales, sociales, por un lado, y los espirituales, subjetivos, individuales, de modo que son primarios los primeros (Celeiro, 2013).

Un fundamento necesario lo constituye la noción teórica de la formación moral y la formación ética de la postura de Celeiro (2013) que permite reconocer el proceso de formación ético-jurídica ambiental del estudiante de la carrera de Derecho como un proceso en el que se entretejen armónicamente los rasgos y las peculiaridades de la formación moral y la formación ética del futuro operador del derecho desde su modo de actuación que posibilita la apropiación de las lógicas conexiones y distinciones que entre estos aspectos existen y su vínculo esencial con una cultura jurídica ambiental, que a partir de estas relaciones se van construyendo.

En lo valorado, la comprensión teórica de la ética ecológica de Leopold (2004), quien concibe desde su posición que la agricultura como cuidado de la tierra y en su "ética de la tierra" contribuye significativamente a esclarecer el desafío de integrar la ética ambiental dentro la práctica científica y enseñanza de la ecología. La comprensión aportada constituye una filosofía ambiental sobre la reconexión, no solo con la diversidad de seres vivos, sino también con la diversidad de pueblos y sus saberes, puesto que, tal como las ciencias ecológicas, estos expresan una íntima conexión entre las vidas e identidades humanas y la tierra donde cohabitamos (Rozzi, 2001). 
Por consiguiente, de acuerdo con la posición de Leopold (2004), una ética de la tierra no puede evitar la alteración, el manejo y el empleo de los recursos naturales, pero sí afirma su derecho a su continua existencia y, por lo menos en ciertos lugares, a que su existencia continúe en un estado natural. En suma, una ética de la tierra cambia el papel del Homo sapiens: de conquistador de la comunidad de la tierra al de simple miembro y ciudadano de ella. Esto implica el respeto por sus compañeros-miembros y también el respeto por la comunidad como tal.

Muchas de las decisiones respecto del uso de la tierra y la biodiversidad están amparadas por intereses y razones económicos, algo que se pondera de manera no adecuada en los actores económicos en el sector agropecuario, aunque en materia de derecho de la propiedad industrial no preconicen de manera adecuada el registro de patentes de invención, el uso de la marca y el lema comercial, el uso de la ecoetiqueta, la ecotributación, como no implementar la publicidad con el empleo de las tecnologías de la información y de la comunicación (TIC), al no existir una formación jurídica por su transversalidad y educación ambiental por su pertinencia en temas de tal relevancia para el comercio interno y el foráneo.

Esta postura fundamenta la pertinencia de integrar el problema ambiental, que abarca lo transversal e intertransversal con la articulación de los saberes. Son retos educativos presentes como realidades sociales en el contexto formativo desde la dimensión ambiental con la que los autores de esta investigación se afilian, por ser más integradora con la relación sociedad-naturaleza y la conformación de una ética comprometida con la preservación ambiental incorporada al individuo, agravada con el cambio climático, en las que son necesarios los aportes de la ciencia para su mitigación desde estudios integradores por la multidimensionalidad de la cuestión ambiental.

Novo (2009), en este sentido, refiere que los problemas como el cambio climático están siendo relegados a un segundo plano, debido, entre otras causas, a la crisis económica, en una estrecha visión que oculta la gravedad del cambio global que está experimentando el planeta. Por ello, las políticas públicas en este sentido van a potenciar el desarrollo, que primero se llamó ecodesarrollo, después desarrollo endógeno y más tarde desarrollo sostenible.

Desde lo jurídico, se significa la pertinencia del principio de prevención, principio rector del derecho ambiental, reforzado con el precautorio y el de legalidad, por cuya existencia se connota lo que se denomina daño permisible, y se precisa a través de él hasta dónde se puede permitir el daño ambiental. Las bases jurídicas de 
la concepción pedagógica que se presenta se erigen en los fundamentos del derecho ambiental y constituyen un llamado a la toma de conciencia sobre la emergencia ecológica en la articulación de un proceso normativo e institucional que desde un aparato legislativo establece como requisito el control público ambiental con la inspección y la auditoría (herramientas de gestión) a partir del derecho público y la pertinencia de que se regule en el ordenamiento jurídico con una ley de educación (Antúnez, 2018).

Por ello, comprender las bases jurídicas en el contexto de la educación ambiental significa reconocer la necesidad de la implementación de las herramientas de gestión como materialización del principio de prevención con el objetivo de incorporar a los proyectos de formación del estudiante de la carrera de Derecho desde su ejercicio profesional. El principio de prevención es el más significativo dentro de los principios ambientales en los ordenamientos jurídicos; claro que están presentes junto a este el precautorio y el de legalidad (Cafferatta, 2004; Antúnez, 2018).

En este contexto, para Berriz (1999), la educación ambiental se considera un tema transversal asociado a todas las áreas del conocimiento que requiere un enfoque globalizador e interdisciplinario. Es un movimiento innovador cuyos principios afectan a todos los componentes del proceso pedagógico, que se refiere a problemas que rebasan el ámbito del sistema educativo y que afectan a toda la sociedad y es esencial en la formación de valores requeridos para la conducta ciudadana.

Se justiprecia que el sustento jurídico-ambiental de la formación del estudiante de la carrera de Derecho favorece la interpretación de la educación ambiental como la herramienta que tributa a la preparación de los operadores del derecho en la protección del entorno desde su modo de su actuación, necesitada de su regulación normativa por el Ministerio de Educación Superior en el ordenamiento jurídico cubano, para que se tracen las pautas a seguir en este sentido.

La educación jurídico-ambiental como dimensión de la educación ambiental es un proceso continuo y permanente orientado al conocimiento generalizado de los derechos y de las obligaciones que, en el proceso de construcción y producción de conocimientos, de desarrollo de hábitos, habilidades y actitudes, así como en la formación de valores jurídicos, armoniza las relaciones entre los seres humanos, y de ellos con el resto de la sociedad y la naturaleza, sobre la base de las categorías de lo justo y lo injusto, lo legal e ilegal, lo legítimo o no que pueden ser estas relaciones en un contexto histórico determinado, para propiciar la orientación de los 
procesos económicos, sociales y culturales hacia el desarrollo sostenible (Álvarez y Vega-Marcote, 2009).

La educación jurídico-ambiental busca la formación de tal cultura, sin embargo, las ciencias de la educación no se han ocupado de este aspecto en profundidad de manera adecuada; no obstante, sobre cultura jurídica o cultura de la legalidad se ha particularizado el conocimiento de los derechos y de las obligaciones, de las prácticas y costumbres a las que se les atribuyen en algunos sistemas de derecho una función razonable y relevante en la vida colectiva, que permita dar cumplimiento adecuado al principio de legalidad (Escasena, 1990).

La educación jurídico-ambiental sobre el derecho ambiental en el contexto de América Latina se reconoce como un entramado complejo de doctrinas, que se va constituyendo en un conjunto de derechos que atraviesa todo el sistema jurídico, en su racionalidad formal, en sus bases materiales, en sus principios axiológicos y en sus instrumentos normativos. Por esta razón, demanda una transformación profunda del régimen jurídico que sostiene a la racionalidad económica dominante e implica una movilización de la verdad que fragua en nuevos fundamentos y formas jurídicas (Almenara, 2001). Posición a la que nos afiliamos por ser pertinente en esta investigación en la actualización del modelo económico y social en relación con la renovación normativa del ordenamiento jurídico cubano a partir de la aprobación del nuevo texto político constitucional.

Se coincide con Cafferatta (2004) al considerar que el derecho ambiental

reclama nuevos métodos, nuevas formas, nuevos criterios de justicia, nuevas leyes [...] para resolver el problema que afrontamos; estos criterios saldrán de una interdisciplinariedad científica que permita definir el quantum desde el punto de vista de las ciencias exactas y naturales, para que el jurista pueda -inmerso en esa realidad - resolver "lo justo de cada uno". (p. 79)

Es preciso sentar posición por los autores del artículo que la incorporación de la dimensión ambiental constituye un recurso metodológico que permite la integración al proceso formativo, un sistema de elementos ambientales (conocimientos, habilidades, actitudes, aptitudes y valores), conscientemente diseñados y contextualizados, que, en la formación de profesionales, debe efectuarse sobre la base de los objetivos generales del modelo del profesional y concretarse en los objetivos de cada año, 
componentes y disciplinas, así como en las diferentes actividades, para contribuir a la formación de un profesional capaz de transformar su entorno.

En este sentido, se pondera que la formación jurídico-ambiental puede ser potenciada a través del turismo histórico de naturaleza, en la interacción en la Sala de lo Económico del Tribunal Popular Provincial en que se analizan los conflictos ambientales, en proyectos de investigación agroambientales y en los procesos de formación de ingenieros agrónomos y médicos veterinarios con una visión desde la teoría-praxis en la formación jurídico-ambiental y de otras carreras.

La sistematización de las normas legales que articulan los diferentes instrumentos jurídico-ambientales favorece el conocimiento general de los derechos y de las obligaciones que, en el proceso de construcción y producción de conocimientos, de desarrollo de hábitos, habilidades y actitudes, así como en la formación de valores jurídicos, armonizan las relaciones entre los seres humanos, y con el resto de la sociedad y la naturaleza, sobre la base de las categorías de lo justo y lo injusto, lo legal e ilegal, lo legítimo o no, para propiciar la orientación hacia el desarrollo sostenible a partir de valores a formar, como la justicia, la responsabilidad, la solidaridad, la disciplina y el rigor profesional, el humanismo y la honestidad en el estudiante de la carrera de Derecho, que desde el Plan E se establecen en Cuba.

Se valora con ello que la educación jurídico-ambiental debe ser vista como un proceso educativo que integra el contenido de las categorías jurídicas relacionadas con el medio ambiente, en el que se establece una dialéctica entre deberes y derechos generadoras de un conocimiento jurídico del estudiante de la carrera de Derecho, que le permite sensibilizarse ante los problemas ambientales y adoptar las herramientas para cumplir y hacer cumplir lo regulado en el ordenamiento jurídico como un profesional con competencias, cuyos modos de actuación se correspondan con el desarrollo de la educación científica del siglo xxi a partir del cumplimiento de la legalidad como principio (Antúnez y Rondón, 2016).

\section{La formación por competencias en el estudiante de la carrera de Derecho}

En consecuencia, la formación por competencias en el campo de lo ambiental deberá, necesariamente, partir de aspectos teóricos conceptuales y terminológicos específicos, sobre la base de programas multidisciplinarios integrados para estar 
en condiciones de participar de manera activa en las decisiones profesionales, por lo que es necesario tener algo que decir, voluntad de hacerlo, medios necesarios, información adecuada, conocimientos sólidos y profunda responsabilidad. Estas condiciones y decisiones deben ser integrales, y estar ajustadas a diferentes niveles; es lo que se espera fomentar en la empresa pública cubana en el sector agropecuario, a partir de ponderarse los principios de seguridad alimentaria y agricultura sostenible con el fomento de la empresa de alta tecnología en el nuevo modelo económico a través del uso de tecnologías limpias para la obtención de producciones no contaminantes al medio ambiente (Antúnez, 2018).

Aunque en el siglo xxi pueda afirmarse que hay un derecho ambiental latinoamericano, diferente del derecho ambiental de otros contextos, el que lo distingue a partir de su reconocimiento en los textos constitucionales, es la concesión de los derechos que se le dio a la naturaleza en las cartas magnas de Bolivia y Ecuador como sujeto dentro del pluralismo jurídico de iure. Estas garantizan a los pueblos indígenas la conservación y el desarrollo de sus formas tradicionales de convivencia y organización social, lo que constituye una expresión de la interculturalidad en el pluralismo jurídico, tema pertinente en la formación jurídico-ambiental como nuevo saber ambiental (Antúnez y Díaz, 2018).

Peña (2016) significa que la constante en los sistemas jurídicos es que todavía no hay un reconocimiento expreso del estatus de sujeto de derecho a la naturaleza, que consiste en que cualquier persona pueda ejercer su representación adecuada a través de esquemas de legitimación procesal amplia en defensa de intereses supraindividuales y, a la vez, pueda solicitar a través de la pretensión procesal la conservación y recomposición del ambiente. Desde una óptica estrictamente jurídica, los derechos de la naturaleza no representan un avance sustantivo, ni procesal, para la evolución del derecho ambiental contemporáneo.

A partir de la postura anterior, se razona que la clave del éxito del acuerdo tomado en París en 2017 residirá en la implicación de toda la comunidad internacional como un ejemplo de gobernanza mundial y en el fomento de concienciación de la sociedad de la necesidad de avanzar hacia un desarrollo sostenible con nuevos modelos de comportamiento y convivencia, para lo cual será necesario seguir profundizando en la educación ambiental. Cuestión con la que se coincide en seguir realizando acciones vinculadas a la formación jurídico-ambiental.

A nivel global y regional, como novedad jurídica, hay una revolución en materia de derechos humanos ambientales y de derechos de la naturaleza. En consecuencia, 
Emmanuel Macron presentó a la Asamblea General de las Naciones Unidas el borrador del Pacto Mundial por el Medio Ambiente (2017), documento que tiene como meta convertirse en el tercer Pacto Internacional de Naciones Unidas, y complementar el de los derechos civiles y políticos, y el de los derechos económicos, sociales y culturales. Algo pertinente para las acciones de educación ambiental en los procesos de formación jurídico-ambiental.

Desde la educación ambiental, se parte del reconocimiento de la educación ambiental como proceso formativo e integrador y de la dimensión del proceso docente educativo. Se estructura en una organicidad interna y en una dirección coherente en la que participan todos los sujetos implicados en el proceso de formación jurídico-ambiental del estudiante de la carrera de Derecho en Cuba, y en el que las influencias educativas solo adquieren verdadero significado cuando son asumidas por él, cuando alcanzan un auténtico sentido y valor individual, y se incorporan como rasgos o cualidades de su personalidad que regulan su comportamiento y posibilitan que este pueda operar con los saberes adquiridos, por lo que su resultado se concreta en la formación ambiental del futuro jurista en su modo de actuación en el escenario que interactúe.

La educación ambiental constituye un aspecto de la formación integral del alumno, de ahí su carácter de dimensión. Sus contenidos están de manera implícita o explícita en los diferentes aspectos que conforman la formación integral, por ello, además, se considera que tiene un carácter multidimensional en el que se articulan los saberes ambientales.

Se asumen los postulados de Lavígne (2007) referidos a la formación ambiental como resultado de un proceso de adquisición de conocimientos, desarrollo de habilidades, hábitos de conductas y apropiación de valores, que comprende la formación de actitudes ambientales, y se expresan en el comportamiento social del alumno en los diferentes espacios de la vida cotidiana.

Se toma la noción de formación ambientalista de Espinoza y Pérez (2011) al reconocer desde su posición que la educación ambiental es un problema social, de naturaleza cognitiva que, por tanto, remite a la apropiación de lo ambientalista por los estudiantes desde su proceso formativo, y transversalizado por la sostenibilidad del desarrollo desde una visión ética que implica compromiso y responsabilidad social en relación con la naturaleza, con su explotación solidaria.

Está claro que la visión para 2020 recogida en Educación superior en el siglo XXI: líneas estratégicas de desarrollo señala que las instituciones de educación superior 
(IES) deben haber "incorporado a los programas docentes elementos formativos orientados a fortalecer la comprensión del entorno social y natural, el desarrollo sustentable, los derechos humanos, la educación para la democracia, la cultura para la paz y la tolerancia" (Asociación Nacional de Universidades e Instituciones de Educación Superior [Anuies], 2000, p. 67).

La cultura ambiental, como expresión concentrada de la actividad humana en relación con el medio ambiente natural o construido, constituye una preocupación de la educación superior, aun cuando no necesariamente encauce sus procesos formativos con la intencionalidad de predeterminar al sujeto con una educación en valores de respeto al medio ambiente, aunque de forma espontánea ha venido cimentándose este valor a partir de la formación de habilidades y procedimientos interpretativos en relación con el medio ambiente, ante la ausencia de un cuerpo legal desde el organismo que así lo disponga.

En la educación ambiental, la noción de incompletud que se aporta desde la ecología de saberes es sumamente relevante, e implica asumir que la educación ambiental nace de las entrañas mismas de la crisis ambiental y se deberá "mover", en el sentido de construir conocimiento, en un contexto cultural caracterizado por el fin de las certidumbres y la aceleración de los tiempos de duración del conocimiento. Lo digital es el entorno por excelencia en el que el conocimiento actual se crea y se distribuye, y permite un crecimiento exponencial de información al irradiarse.

Para Leff (2001), la formación ambiental debe rescatar los saberes ancestrales, integrar las prácticas tradicionales con las prácticas tecnológicas modernas para proyectar acciones de mejoramiento o solución de los problemas ambientales en las comunidades, determinar sus causas y posibles alternativas de solución, y devolver a ellas el saber generado para su aplicación a través de proyectos y programas de gestión ambiental. El saber ambiental problematiza el conocimiento fraccionado en disciplinas y la administración sectorial del desarrollo, para constituir un campo de conocimientos teóricos y prácticos orientado hacia la rearticulación de las relaciones sociedad-naturaleza. Asimismo desborda a las ciencias ambientales constituidas como un conjunto de especializaciones surgidas de la incorporación de los enfoques ecológicos a las disciplinas tradicionales — antropología ecológica, ecología urbana, salud, psicología, economía, ingeniería ambiental- y se extiende más allá del campo de articulación de las ciencias.

Desde estas concepciones, la formación ambiental debe estar orientada a superar la especialización y fragmentación del conocimiento en disciplinas, de manera que 
promueva una visión más integradora del mundo, abierta a la complejidad de los fenómenos de la realidad, y a través del cual resulte este saber emergente en estrecho vínculo con los problemas ambientales. Sin embargo, en la práctica educativa, ha prevalecido una especialización y fragmentación del conocimiento en disciplinas; se produce una jerarquización de información en detrimento del desarrollo de habilidades, que ha permitido, en el mejor de los casos, mitigar o solucionar problemas ambientales desde cada campo del saber, pero que ha impedido una visión holística, integrada a la actuación del profesional.

\section{El uso de las herramientas tecnológicas de la web 2.0 en las competencias laborales e investigativas del estudiante de la carrera de Derecho}

Una propuesta de enseñanza innovadora es la implementación de las herramientas tecnológicas de la web 2.0, las que son implementadas para fortalecer la educación ambiental y su mejor comprensión, en que, a través de estos recursos tecnológicos, se pretende mejorar el aprendizaje en los educandos con la utilización de las Tic en los nuevos escenarios educativos. Su incorporación es sinónimo de modernización, calidad, productividad, mejores servicios y apoyo a los procesos educativos; en la educación, surge la necesidad cada vez mayor del uso de la información en las instituciones de la educación superior.

Las TIC forman parte fundamental de la sociedad del conocimiento. Su uso ha propiciado cambios en el modo en que las personas trabajan, se relacionan y aprenden. Conforman entornos de aprendizaje novedosos que brindan nuevas formas de leer y escribir, de aprender y de enseñar en los actuales escenarios educativos universitarios mediados por las tecnologías (Pérez, Barragán y Molina, 2017).

Al respecto, Cabrero (2000) señala la fortaleza que tiene integrar la educación ambiental a las TIC, e indica que estas herramientas nos ofrecen nuevas formas de desarrollar el aprendizaje, lo que permitirá que el alumno posea experiencias y escenarios que le faciliten su propia construcción del conocimiento, usarlas como medio de búsqueda, comunicación, participación y expresión.

Lozano (2011) considera que las TIC pueden ser utilizadas como herramientas didácticas al servicio del aprendizaje, por lo que se convierten en recursos del aprendizaje y del conocimiento para el estudiante. En este sentido, la implementación de las Tic en la enseñanza de la educación ambiental tiene un papel primordial en 
los escenarios educativos, puesto que implica la incorporación de estas tecnologías al proceso de enseñar el conocimiento complejo. Pero en tanto la complejidad es esencia y condición constitutiva de las interacciones recíprocas entre la naturaleza y la sociedad, los entornos virtuales, lejos de extrañarnos o alejarnos de esa realidad, deben servirnos para volver a ver "el lugar donde habitamos" con otros ojos (Corbetta, 2015).

La educación ambiental y las Tic complementan los elementos teórico-prácticos y desarrollan en el educando habilidades, destrezas y conductas que propician que el alumno haga suyos los conocimientos sobre el medio ambiente, la sustentabilidad, su uso en beneficio del ambiente, descubra nuevas herramientas que le permitirán contribuir al mejoramiento de la relación hombre-naturaleza, lo que consecuentemente formará ciudadanos más responsables y conscientes del cuidado de la naturaleza. Para lograr una educación ambiental sustentable con el uso de las TIC, será necesario conocerlas, a fin de usarlas en beneficio y alcanzar mayor cultura ambiental.

En este orden, en la actual sociedad del conocimiento, el sistema educativo debe favorecer la formación continua y permanente de los docentes de la educación superior, a fin de responder a las necesidades, los intereses y los retos del estudiante. Para lograrlo, será necesario facilitar el acceso a internet en cualquier sitio, a fin de promover el aprendizaje y la formación mediada por tecnologías. Será necesario fortalecer un aprendizaje continuo a lo largo de la vida del profesorado y profesionales, para potenciar competencias cognitivas, procedimentales y actitudinales ante los avances tecnológicos.

Cabero y Llorente (2005) informan que, entre las palabras que han prevalecido a finales del siglo xx y a comienzos del siglo xxi, se encuentra la de "medio ambiente" y “tecnologías de la información”. El cuidado y respecto por el medio ambiente se ha convertido en un valor significativo en la sociedad, que ha impregnado sus sistemas educativos, y ello posiblemente como consecuencia del aumento demográfico, del imparable consumismo, del agotamiento de recursos que parecían inagotables, del aumento exponencial de los residuos, de la imparable contaminación y de las transformaciones en los valores que se están produciendo en la esfera humana. Ya en la Carta de Belgrado formulada en 1975, se hablaba de que el individuo debería ser formado en un mínimo de competencias y capacidades ambientales.

Murga-Menoyo y Novo-Villaverde (2014) afirman que, dentro de las investigaciones que se han realizado en el campo de las TIC y la educación ambiental, se 
encuentra la de mapas conceptuales con CmapTools en la enseñanza universitaria de la educación ambiental. Se notifica una experiencia exitosa en la Universidad Nacional de Educación a Distancia (UNED), en dos materias de la Licenciatura de Pedagogía que imparte la Facultad de Educación: Educación Ambiental y Desarrollo Sostenible, sus implicaciones sociales y educativas.

Cabero et al. (2006) afirman la necesidad de la formación de las nuevas generaciones en lo referente al cuidado de medio ambiente, por lo que recomiendan la inclusión de esta temática en el currículo. Plantean que es necesario formar individuos responsables y conscientes del cuidado del medio ambiente en pro de una explotación racional de los recursos naturales, que es indispensable para que la sociedad futura pueda encontrar el equilibrio natural y entonces sí poder hablar de sociedades sustentables que vivan en armonía con la naturaleza y sus recursos. Los autores señalan que el uso de tecnología favorece y es de ayuda para gestionar las actividades académicas, lo cual requiere un adiestramiento y capacitación docente.

Algunos de los recursos tecnológicos que pueden ser utilizados en la formación ambiental son el video educativo, el multimedia, los blogs, las wikis, las bibliotecas virtuales e internet. En la web 2.0, podemos encontrar aplicaciones que pueden contribuir a la irradiación de la educación ambiental con el uso de plataformas como Facebook, Twitter, LinkedIn, YouTube, entre otras, las cuales abren canales interesantes en relación con la cuestión ambiental. Se reflexiona, en este sentido, que será necesario dar paso a modelos pedagógicos innovadores, con la fortaleza de la utilización de las redes sociales en los escenarios educativos, para crear, organizar y compartir contenidos en el tema relacionado con la educación ambiental, y así gestionar su propio aprendizaje a los estudiantes (Soler del Sol, 2013).

Desde lo sociológico, son asumidos los fundamentos de la sociología de la educación en la que se explica la educación como función de la sociedad, y se enmarca el papel de la educación como institución social en la que tiene lugar la formación del profesional. Los postulados de esta naturaleza ubican a la universidad como la institución escolar en un lugar central en los procesos de educación socialmente institucionalizados como un sistema de influencias sobre los procesos de asimilación con carácter intencional, sistemático y especializado. De manera particular, la concepción de las funciones formativas del educador, enfocadas en la perspectiva de sus tareas básicas, están centralizadas en dos grandes campos: la instrucción y la educación, en tanto el proceso de formación que se concibe en el contexto universitario parte de la dialéctica de lo instructivo y lo educativo mediado por lo orientador, tal y como se concibe en esta investigación. 
Desde lo psicopedagógico, son refrendados los postulados del enfoque socihistórico cultural de Vygotski (1987) y sus continuadores, al reconocer que las funciones psíquicas superiores son un producto de la apropiación de la experiencia histórico-social y se encuentran en los objetos y fenómenos que, a su vez, constituyen síntesis del decursar histórico de la humanidad, es decir, la apropiación constituye el mecanismo fundamental por el cual se produce el desarrollo psíquico humano. Según esta teoría, las estructuras de la mente se forman como producto de la apropiación del bagaje cultural producido por la evolución histórica de la humanidad que se transmite en la relación educativa, las cuales se comunican de generación en generación e implican contenidos, conocimientos, junto con formas, estrategias, modelos de conocimiento, que el individuo capta, comprende, asimila y practica; por esto, se resalta el valor de la instrucción y se le da gran peso a la actividad tutorada (Rodríguez, 2014).

Del modelo de orientación educativa, Fernández (1999) asume los postulados que refrendan la orientación desde su función preventiva como una relación de ayuda que implica el desarrollo de las potencialidades de cada sujeto a través del diálogo y de un amplio proceso interactivo. Desde su reconocimiento sobre la orientación educativa como un proceso con carácter estratégico, sistémico, metodológico y educativo, se significa su sentido potenciador en el desarrollo de la subjetividad del futuro operador del derecho y particularmente en la apropiación de los contenidos jurídico-ambientales, a través del proceso interactivo que tiene lugar en el contexto de su formación inicial.

Se reconoce el proceso de formación jurídico-ambiental del estudiante de la carrera de Derecho como un tipo especial de orientación educativa encaminada a desarrollar los recursos personales del sujeto como herramientas para la comprensión del sentido jurídico-ambiental del ejercicio profesional del jurista desde su modo de actuación como juez, fiscal, abogado, asesor jurídico, consultor jurídico o como profesional (Patarroyo y Benavides, 2014).

El enfoque desarrollador del aprendizaje elaborado y desarrollado por Castellanos et al. (2002) concibe el aprendizaje como un proceso que ocurre a lo largo de toda la vida y que se da en diferentes espacios. En él se cristaliza la dialéctica entre lo histórico-social y lo individual-personal que supone el tránsito de lo externo a lo interno, de lo inter a lo intrapsicológico, de la regulación externa a la autorregulación. Posee un carácter intelectual y emocional, y es un proceso de participación, colaboración e interacción. 
Se pondera que estos fundamentos son pertinentes y permiten en la concepción que se presenta cualificar y caracterizar el aprendizaje jurídico-ambiental del profesional como desarrollador desde su modo de actuación. Este fundamento pedagógico general se concreta en la postura de Álvarez y Vega-Marcote (2009) que refrenda a la pedagogía como ciencia que estudia la formación, en general, y que, por tanto, permite dirigir científicamente este proceso para alcanzar altos niveles de calidad y excelencia.

Al respecto, Miranda (2014) planteó que los problemas ambientales constituyen la base para el desarrollo de la educación ambiental en el proceso de enseñanza-aprendizaje, pues es en función de su expresión en la comunidad, a partir del principio de pensar en lo global y actuar en lo local, que se deben planificar las distintas actividades en la escuela, así como vincular el contenido de las asignaturas y lograr la participación activa de los estudiantes para su prevención o mitigación (Pardo, 2014).

En consecuencia, se justiprecia que la concepción pedagógica de la formación jurídico-ambiental del estudiante de la carrera de Derecho se estructura como un todo, cada componente se interrelaciona con el otro en la expresión dialéctica de lo general y lo singular a través de lo particular y de las relaciones entre el todo y las partes, lo que permitirá conformar el modelo pedagógico de formación ambiental con competencias desde su modo de actuación en el escenario que le corresponda interactuar.

\section{Conclusiones}

Desde la Conferencia Intergubernamental sobre Educación Ambiental, Tbilisi, URss, 14-26 de octubre de 1977, la formación ambiental fue concebida junto con la educación como un proceso de constitución de un saber interdisciplinario y de nuevos métodos holísticos para analizar los complejos procesos socioambientales que emergen del cambio global (Decenio de las Naciones Unidas de la Educación para el Desarrollo Sostenible (2005-2014): plan de aplicación internacional). Todo lo cual constituye un importante reto a enfrentar por la universidad en su misión de formar profesionales.

En los aspectos cognitivos, afectivos y conductuales que constituyen la formación jurídico-ambiental que se identifican en el proceso como la educación ambiental y la norma jurídico-ambiental, para esta integración el docente debe tener 
en cuenta los elementos esenciales que definen la lógica interna de la formación jurídico-ambiental del estudiante de la carrera de Derecho y construir las situaciones de aprendizaje jurídico-ambiental.

El desarrollo de un aprendizaje jurídico-ambiental por parte del estudiante de la carrera de Derecho de los aspectos básicos para un adecuado comportamiento ambiental se ejecuta a través del planteamiento de situaciones que viabilizan la práctica de una participación activa, crítica y reflexiva sobre la base de los valores de una cultura ambiental que tiene su reflejo en las actitudes ambientales para ser un mejor profesional.

Las situaciones de aprendizaje desde la problematización se generan a partir de un planteamiento heurístico y creativo, en la elaboración de los proyectos educativos integradores. La apropiación del problema ambiental se manifiesta en la medida en que el estudiante de la carrera de Derecho es capaz de realizar acciones en torno a los conocimientos que va adquiriendo en coherencia con sus pensamientos y sentimientos, desde la perspectiva del cuidado, la protección y el desarrollo del medio ambiente, al tener en cuenta el carácter complejo e integrador de este mediado con el uso de las Tic, con lo cual hace un profesional con competencias.

En Cuba, no existen los estudios de carreras ambientales, por lo que esta discusión académica ha sido parte de la política de trabajo del Programa de Medio Ambiente del Ministerio de Educación Superior sin respuesta. Para ello, se trabaja en el carácter transversal el problema ambiental en los procesos sustantivos de la universidad: docencia, investigación y extensión.

\section{Referencias}

Álvarez Suárez, P. y Vega-Marcote, P. (2009). Actitudes ambientales y conductas sostenibles: implicaciones para la educación ambiental. Revista de Psicodidáctica, 14, 25-38.

Álvarez de Zayas, C. (1995). La escuela en la vida. Sucre, Bolivia: Universidad San Francisco Javier.

Antúnez Sánchez, A. (2018). La formación ambiental: una necesidad en el siglo xxi con el nuevo saber ambiental. Revista Murmullos del Sur, 34(2), 89-124. 
Antúnez Sánchez, A. y Díaz Ocampo, E. (2018). La hipercultura en la formación jurídica. Urbe et Ius. Recuperado de http://urbeetius.org/la-hipercultura-en-la-formacion-juridica/

Antúnez Sánchez, A. y Rondón Valdés, J. (2016). La formación de habilidades en estudiantes de Derecho, una tarea de formar no de informar. Apuntes de Ciencia \& Sociedad, 6(2), 150-157.

Asociación Nacional de Universidades e Instituciones de Educación Superior (2000). La educación superior en el siglo XXI. Lineas estratégicas de desarrollo: una propuesta de la Anuies. La Habana, Cuba: Autor.

Ayes Ametller, G. N. (2013). Medio ambiente: impacto y desarrollo. La Habana, Cuba: Editorial Científico-Técnica.

Badillo Mendoza, M. E. (2015). Prácticas contrainformativas como expresión de la ciberciudadanía ambiental. Madrid, España: Universidad Nacional de Educación a Distancia.

Barragán Ronderos, D. (2016). La construcción de la mentalidad democrática como necesidad en el posconflicto. Via Inveniendi et Iudicandi, 11(1), 37-57. DOI: https:// doi.org/10.15332/s1909-0528.2016.0001.06

Bayón Martínez, P. (2006). Educación ambiental, participación y transformación social sostenible en Cuba. Revista Interface, 2, 89-104.

Bermúdez Sarguera, C. R. y Rodríguez Rebustillo, C. M. (2003). La estructura de la actividad propuesta por A.N. Leontiev pudiera ser psicológicamente inconsistente. Revista Cubana de Psicología, 20(1), 80-87.

Berriz, A. (1999). La educación ambiental. Madrid, España: Centro de Estudios Políticos y Constitucionales.

Bravo Mercado, M. T. (1997). Dimensión ambiental y currículum universitario: el currículum universitario de cara al nuevo milenio. Ciudad de México, México: Universidad Nacional Autónoma de México. 
Cabero Almenara, J. (1998). Impacto de las nuevas tecnologías de la información y la comunicación en las organizaciones educativas. En M. Lorenzo Delgado, J. A. Ortega Carrillo y T. Sola Martínez (coords.), Enfoques en la organización y dirección de instituciones educativas formales y no formales (pp. 197-206). Granada, España: Grupo Editorial Universitario.

Cabero Almenara, J. (2000). La utilización educativa de la televisión y el video. En Nuevas tecnologías aplicadas a la educación (pp. 39-70). Madrid, España: Síntesis.

Cabero Almenara, J. (2001). Tecnología educativa: diseño, producción y evaluación de medios. Barcelona, España: Paidós.

Cabero Almenara, J. (2004). Formación del profesorado en Tic: el gran caballo de batalla. Comunicación y Pedagogía, 195, 27-31.

Cabero Almenara, J. y Llorente Cejudo, M. del C. (2005). Las Tic y la educación ambiental. Revista Latinoamericana de Tecnología Educativa, 4, 9-26.

Cabero, J., Morales, J. A., Romero, R., Barroso, J., Castaño, C., Román, P. ... Salinas, J. (2006). Formación del profesorado universitario en estrategias metodológicas para la incorporación del aprendizaje en red en el Espacio Europeo de Educación Superior (EEES). Píxel-Bit, Revista de Medios y Educación, 27, 11-19. Recuperado de https:// www.redalyc.org/pdf/368/36802702.pdf

Cafferatta, N. (2004). Constitucionalismo e institucionalidad ambiental en Latinoamérica. Ciudad de México, México: Instituto Nacional de Ecología.

Canes Garrido, F. (1995). Antecedentes históricos de la educación ambiental: la Antigüedad clásica. Revista Complutense de Educación, 6(2), 29-75.

Casas, M. (2001). La formación ambiental en las carreras económicas (Tesis doctoral, Universidad de Pinar del Río, Cuba).

Castellanos Simons, D., Castellanos Simons, B., Llivina Lavigne, M. J., Silverio Gómez, M., Reinoso Cápiro, C. y García Sánchez, C. (2002). Aprender y enseñar en la escuela: una concepción desarrolladora. La Habana, Cuba: Editorial Pueblo y Educación. 
Celeiro Carbonell, A. (2013). La cultura ético-axiológica humanista del profesional de la educación desde la formación inicial (Tesis doctoral, Universidad de Ciencias Pedagógicas Frank País García, Santiago de Cuba, Cuba).

Comisión Económica para América Latina y el Caribe (2016). Agenda 2030 y los Objetivos de Desarrollo Sostenible: una oportunidad para América Latina y el Caribe. Santiago de Chile, Chile: Autor.

Corbetta, S. (2015). Pensamiento ambiental latinoamericano y educación ambiental. Revista del Plan Fénix. Voces en el Fénix. Voces de la Tierra, 6(43).

Coya García, M. (2001). La ambientalización de la universidad: un estudio sobre la formación ambiental de los estudiantes de la Universidad de Santiago de Compostela y la política ambiental de la institución (Tesis doctoral, Universidad de Santiago de Compostela, Santiago de Compostela, España).

Decenio de las Naciones Unidas de la Educación para el Desarrollo Sostenible (2005-2014): plan de aplicación internacional.

Delgado Díaz, C. J. (2006). La educación ambiental desde la perspectiva politica. La Habana, Cuba: José Martí.

Delgado Ramos, G. C., Cornetta, A. y Díaz, B. (2014). Cambio climático global, transformación agraria y soberania alimentaria en América Latina. Buenos Aires, Argentina: Consejo Latinoamericano de Ciencias Sociales.

Díaz Ocampo, E. y Antúnez Sánchez, A. (2018). El pluralismo jurídico en América Latina. Revista dos Tribunais, 107(990), 333-358.

Díaz Ocampo, E., Guanoquiza, L. y Antúnez Sánchez, A. (2018). El uso de las infotecnologías en la formación ambiental del ciudadano. Revista Derecho y Cambio Social, 52. Recuperado de https://www.derechoycambiosocial.com/revista052/EL_USO_DE_ LAS_INFOTECNOLOGIAS.pdf 
Díaz Rodríguez, S. N. (2011). Sistema de procedimientos metodológicos para la formación del pensamiento iusflosófico (Tesis doctoral, Centro de Estudios de Educación Superior Manuel F. Gran. Universidad de Oriente, Santiago de Cuba, Cuba).

Domingos Víctor, J., Díaz Rodríguez, S. N. y Antúnez Sánchez, A. (2014). La formación de la responsabilidad ambiental en los profesores que imparten Derecho del Medio Ambiente en la carrera de Derecho en la educación superior en Angola. Pensamiento Jurídico, 40, 137-158.

Fernández, S. (2003). Desarrollo de competencias: ¿̨de quién o de qué dependen? Ciudad México, México: Mercer Human Resource Consulting.

Escasena, J. (1990). La evolución de la legalidad en Cuba La Habana, Cuba: Editorial Ciencias Sociales.

Eschenhagen, M. L. (2005). Los desafíos de la educación ambiental en épocas del desarrollo sostenible: algunas reflexiones alrededor del desarrollo, la educación ambiental y la interdisciplinariedad. Hombre y Desierto, 12, 73-86.

Eschenhagen, M. L. (2012). Aproximaciones al pensamiento ambiental de Enrique Leff. Environmental Ethics, 34, 89-95.

Espinosa Gallegos-Anda, C. y Pérez Fernández, C. (Eds.) (2011). Los derechos de la naturaleza y la naturaleza de sus derechos. Quito, Ecuador: Ministerio de Justicia, Derechos Humanos y Cultos.

Fernández Rubio-Legrá, Á. (1999). Ley No. 81 de 1997, del Medio Ambiente: en más de 150 preguntas y respuestas. La Habana, Cuba: Ministerio de Justicia.

Galeano, E. (2005). El imperio del consumo. Recuperado de http://latinoamericana.org/2005/ textos/castellano/Galeano.htm

García, J. E. (2002). Los problemas de la educación ambiental: ¡es posible una educación ambiental integradora? Revista Investigación en la Escuela, 46, 5-25.

García Gómez, J. y Nando Rosales, J. (2000). Estrategia didáctica en educación ambiental. Málaga, España: Aljibe. 
García, D. y Priotto, G. (2009). Educación ambiental: aportes políticos y pedagógicos en la construcción del campo de la educación ambiental. Buenos Aires, Argentina: Secretaría de Ambiente y Desarrollo Sustentable de la Nación.

García Díaz, J. E. (2000). Educación ambiental y ambientalización del currículo: didáctica de las ciencias experimentales. En F. J. Perales Palacios y P. Cañal de León (Coords.), Didáctica de las ciencias experimentales: teoría y práctica de la enseñanza de las ciencias (pp. 585-614). Madrid, España: Marfil.

Gaudiano, E. (2005). Educación ambiental. Lisboa, Portugal: Instituto Piaget.

González Apaolaza, R. y Deschamps, M. (2002). Desarrollo de la educación basada en competencias en México. Ciudad de México, México.

González Novo, T. y García Díaz, I. (1998). Cuba, su medio ambiente después del medio milenio. La Habana, Cuba: Editorial Científico-Técnica.

Giuffré, L. (2004). Los objetivos de desarrollo del milenio y la educación ambiental. Revista de la Facultad de Agronomía, 24(2), 133-138.

Holbach, P. H. D. (1989). Sistema de la naturaleza. La Habana, Cuba: Editorial Ciencias Sociales.

Indiana Basterra, N. y Silvana Peralta, E. (2014). Introducción a la educación ambiental: bases para la formación ambiental de los alumnos universitarios. Resistencia, Argentina: Universidad Nacional del Nordeste.

Jiménez Guethón, R. y Antón Torres, N. A. (2014). Agroecología y seguridad alimentaria: una visión desde Cuba. Estudios del Desarrollo Social: Cuba y América Latina, 2(2), 62-76.

Lavígne, M. (2007). La educación ambiental para el desarrollo sostenible. La Habana, Cuba: Editorial Pueblo y Educación.

Leff Zimmerman, E. (2000a). Ambiente, interdisciplinariedad y currículum universitario: la educación superior en la perspectiva de desarrollo sostenible. Formación Ambiental, 9, 9-20. 
Leff Zimmerman, E. (2000b). Saber ambiental. Ciudad de México, México: Siglo xxi.

Leff Zimmerman, E. (2001). Conocimiento y educación ambiental: formación ambiental. Red de Formación Ambiental para América Latina y el Caribe.

Leff Zimmerman, E. (2009). La pedagogía del ambiente: formación ambiental y desarrollo sustentable. Pinar del Río, Cuba.

Leopold, A. (2004). Reporte de psicología. Berlín, Alemania: Charitsd.

Mateo Rodríguez, J. M. (2001). La cultura de la naturaleza como base de la educación ambiental. Anuario de Ecologia, Cultura y Sociedad, 1.

Mateo Rodríguez, J. M. (2003). Pensamiento sostenibilista y educación ambiental: ¿entrecruzamiento o articulación? Trabajo presentado en el IV Congreso Iberoamericano de Educación Ambiental, La Habana, Cuba.

Mateo Rodríguez J. M. y Suárez Gómez, C. (2001). La ciencia y la tecnología en el debate ambiental. La Habana, Cuba: Félix Varela.

Martínez Castillo, R. (2012). Ensayo crítico sobre educación ambiental. Revista Electrónica Diálogos Educativos, 24, 70-104.

Miranda López, A. del C. (2014). Educación ambiental en el proceso de enseñanza-aprendizaje en primaria, secundaria y preuniversitario. Revista Vinculando. Recuperado de http://vinculando.org/ecologia/educacion-ambiental-en-el-proceso-de-ensenanza-aprendizaje-en-primaria-secundaria-y-preuniversitario.html

Molano Niño, A. C. y Herrera Romero, J. F. (2014). La formación ambiental en la educación superior: una revisión necesaria. Revista Luna Azul, 39, 186-206.

Moré, M. (2010). La formación ambiental permanente del directivo educacional del siglo XXI (Tesis doctoral, Universidad de Ciencias Pedagógicas Félix Varela Morales, Santa Clara, Cuba). 
Murga-Menoyo, M. A. y Novo-Villaverde, M. (2014). Sostenibilizar el currículum: la Carta de la Tierra como marco teórico. Edetania: Estudios y propuestas socioeducativas, 46, 163-179.

Novo-Villaverde, M. (1986). Educación y medioambiente. Madrid, España: UnED.

Novo-Villaverde, M. (1995). Educación ambiental y desarrollo sostenible. En, Educación ambiental: cuestiones y propuestas. Murcia, España: Obra Social de Cajamurcia.

Novo-Villaverde, M. (2000). Educación ambiental: teoría y práctica. Revista Iberoamericana de Educación, 11.

Novo-Villaverde, M. (2006). Educación ambiental: desarrollo sostenible y globalización. Revista de Educación Ambiental, 4(6), 24-31.

Novo-Villaverde, M. (2009). La educación ambiental, una genuina educación para el desarrollo sostenible. Revista de Educación, 1, 195-217.

Novo-Villaverde, M. y Lara, R. (1997). El análisis interdisciplinar del problema ambiental. Madrid, España: Fundación Universidad-Empresa.

Novo-Villaverde, M. y Murga, M. Á. (2010). Educación ambiental y ciudadanía planetaria. Revista Eureka sobre Enseñanza y Divulgación de las Ciencias, 7, 179-186.

Núñez Morales, M. (2004). La educación en valores ambientales del estudiante de la carrera de Derecho (Tesis de maestría, Universidad de Camagüey, Camagüey, Cuba).

Ojalvo, V. (2001). La educación de valores en el contexto universitario. La Habana, Cuba: Félix Varela.

Pardo Posada, N. E. (2014). Un recorrido por los derechos colectivos en la jurisprudencia argentina. Via Inveniendi et Iudicandi, 9(1), 32-49. DOI: https://doi.org/10.15332/ s1909-0528.2014.0001.02

Patarroyo Rengifo, S. y Benavides Gómez, P. A. (2014). Rupturas asignificantes: revisiones críticas en torno al Derecho. Via Inveniendi et Iudicandi, 9(1), 7-31. DOI: https://doi. org/10.15332/s1909-0528.2014.0001.01 
Peña Chacón, M. (2016). Derecho ambiental efectivo. San José, Costa Rica: Universidad de Costa Rica.

Pérez-Jorge, D., Barragán-Medero, F. y Molina-Fernández, E. (2017). A study of educational programmes that promote attitude change and values education in Spain. Asian Social Science, 13(7), 112-130.

Rey Santos, O. y Cruz Sardiñas, T. (2017). La Ley del Medio Ambiente: 20 años después. La Habana, Cuba: Unijuris.

Rodríguez Cely, A. M. (2014). Indicadores de constitucionalidad de las políticas públicas: enfoque de gestión de derechos. Via Inveniendi et Iudicandi, 9(2), 135-175. DOI: https:// doi.org/10.15332/s1909-0528.2014.0002.06

Roque, M. (2003). Estrategia educativa para la formación de la cultura ambiental de los profesionales cubanos de nivel superior, orientada al desarrollo sostenible (Tesis doctoral, Universidad de Ciencias Pedagógicas Enrique José Varona, La Habana, Cuba).

Rozzi, S. J. de (2001). Derecho ambiental en el nuevo milenio: una estrategia para el derecho ambiental. Revista de estudios locales: Cunal, 1, 9-14.

Sauvé, L. (2010). Educación científica y educación ambiental: un cruce fecundo. Enseñanza de las Ciencias: Revista de Investigación y Experiencias Didácticas, 28(1), 5-18.

Soler del Sol, A. (2013). El derecho al alcance de todos, Ley No. 81 Ley del Medio Ambiente. La Habana, Cuba: ONBC.

Valdés Valdés, O. (2002). La educación ambiental y la protección del medio ambiente. Revista Educación, 105, 8-15.

Velázquez de Castro, F. (2002). La educación ambiental en el siglo XXI. Granada, España: Grupo Editorial Universitario.

Vygotski, L. S. (1987). Pensamiento y lenguaje: teoría del desarrollo cultural de las funciones psíquicas. La Habana, Cuba: Pueblo y Educación. 